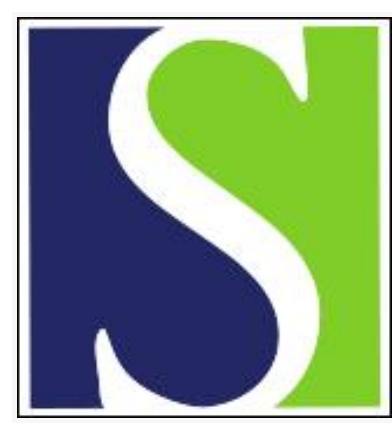

Scand J Work Environ Health 2000;26(3):213-218

https://doi.org/10.5271/sjweh.534

Issue date: Jun 2000

Biological monitoring of $\mathrm{N}$-methyl-2-pyrrolidone using 5-hydroxy-N-methyl-2-pyrrolidone in plasma and urine as the biomarker

by Åkesson B, Jönsson BAG

The following article refers to this text: 2004;30(4):306-312

Key terms: graffiti remover; paint stripper; reproductive toxicity; skin uptake; solvent

This article in PubMed: www.ncbi.nlm.nih.gov/pubmed/10901113

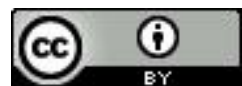




\title{
Biological monitoring of N-methyl-2-pyrrolidone using 5-hydroxy-N-methyl- 2-pyrrolidone in plasma and urine as the biomarker
}

\author{
by Bengt Åkesson, PhD, ${ }^{1}$ Bo AG Jönsson, PhD'
}

\begin{abstract}
Åkesson B, Jönsson BAG. Biological monitoring of N-methyl-2-pyrrolidone using 5-hydroxy-N-methyl-2-pyrrolidone in plasma and urine as the biomarker. Scand J Work Environ Health 2000;26(3):213-218.

Objectives The aims were to study the toxicokinetics of 5-hydroxy-N-methyl-2-pyrrolidone (5-HNMP) in blood and urine after exposure to N-methyl-2-pyrrolidone (NMP) and to study the suitability of 5-HNMP as a biomarker for assessing NMP exposure.

Methods Six male volunteers were exposed for 8 hours to NMP concentrations of $0,10,25$, and $50 \mathrm{mg} / \mathrm{m}^{3}$. Blood and urine were sampled before, during, and up to 40 hours after exposure. Aliquots of urine and plasma were purified, derivatized, and analyzed for 5-HNMP on a gas chromatograph/mass spectrometer in the electron impact mode.

Results The mean plasma concentration [P-(5-HNMP)] after 8-hour NMP exposure to 10,25 , and $50 \mathrm{mg} / \mathrm{m}^{3}$ was $8.0,19.6$, and $44.4 \mu \mathrm{mol} / 1$, respectively. The mean urinary concentration [U-(5-HNMP)] for the 2 last hours of exposure was $17.7,57.3$, and $117.3 \mathrm{mmol} / \mathrm{mol}$ creatinine, respectively. The maximal P-(5-HNMP)and U-(5HNMP) concentrations occurred 1 hour and $0-2$ hours, respectively, after the exposure. The half-times of P-(5HNMP) and U-(5-HNMP) were 6.3 and 7.3 hours, respectively. The 5-HNMP urinary concentrations were $58 \%$ of the calculated retained dose. There was a close correlation ( $r)$ between P-(5-HNMP) $(r=0.98)$ and U-(5-HNMP) $(\mathrm{r}=0.97)$ with NMP exposure.

Conclusions 5-HNMP is an excellent biomarker for assessing exposure to NMP. Its plasma and urinary half-times (6-7 hours), the minimal risk for contamination during sampling in occupational settings, and the close correlation of $\mathrm{P}-(5-\mathrm{HNMP})$ and $\mathrm{U}-(5-\mathrm{HNMP})$ with NMP exposure makes 5-HNMP suitable for monitoring exposure to NMP. 5-HNMP in plasma is recommended.
\end{abstract}

Key terms graffiti remover, paint stripper, reproductive toxicity, skin uptake, solvent.

N-Methyl-2-pyrrolidone (NMP; CAS number 872 -504) is, due to its strong and selective solvent power, a widely used organic solvent. NMP is a water-miscible colorless liquid. It is hygroscopic with a mild amine odor. NMP is used in the petrochemical industry, in the microelectronics fabrication industry, and in the manufacturing of various compounds (eg, pigments, cosmetics, drugs, insecticides, herbicides, and fungicides). NMP is used as a substitute of solvents of higher inherent toxicity in occupational and environmental settings (eg, dichloromethane in paint strippers). The use of NMP as a remover of graffiti has strongly increased. NMP has been suggested as a skin penetration enhancer for use in transdermal therapy in humans (1).

Experimental exposure of human volunteers shows that NMP is readily absorbed through the respiratory tract and eliminated from the body (mainly) by biotransformation to other compounds. Only about $2 \%$ of the inhaled dose is excreted in urine as NMP (2). In the rat, Wells and his co-workers (3) showed that NMP is mainly metabolized to 5-hydroxy-N-methylpyrrolidone (5HNMP). A metabolic pathway in humans, where NMP is first hydroxylized to 5-HNMP and then further oxidized to N-methylsuccinimide (MSI), which in turn is hydrolyzed to 2-hydroxy-N-methylsuccinimide (2HMSI), has been suggested (4). It has been shown that NMP possesses a high permeability through both human (5) and rat $(6,7)$ skin. In rat, about $70 \%$ of the radioactivity of skin-administered radiolabeled NMP is excreted in urine (7).

Animal studies show that exposure to NMP may cause degenerative changes in the respiratory system and in hemopoietic and lymphoid tissues. Lethargy and irregular respiration have also been recorded (8). Studies in female rats exposed to a concentration of $1000 \mathrm{mg} / \mathrm{m}^{3}$ showed only minor nasal irritation when exposed through

Reprint requests to: Dr B Åkesson, Department of Occupational and Environmental Medicine, University Hospital, S-221 85 Lund, Sweden. [E-mail: bengt.akesson@ymed.lu.se] 
the nose only, but massive increased mortality and severe effects on major organs occurred when whole-body exposure was employed (unpublished data).

Experimental NMP exposure of humans for 8 hours to $50 \mathrm{mg} / \mathrm{m}^{3}$ indicates that NMP is a mild irritant (2). However, in occupational settings, an irritative effect has been recorded on skin and eyes, and this finding indicates that NMP may be a moderate to severe irritant $(9$, 10).

Animal studies on reproductive toxicity show that NMP may cause developmental toxicity at doses causing mild or no maternal toxicity (11). Moreover, a stillbirth after occupational exposure to NMP has recently been described in a case report (12).

The large number of women exposed to NMP in industry makes it important to develop methods for assessing exposure to NMP. Methods for analyzing NMP in air have been described (2). However, the extensive percutaneous absorption of NMP makes biological monitoring preferable for assessing the exposure. A method, NMP in plasma and urine, for the biological monitoring of NMP exposure has also been reported (2). However, the ready biotransformation of NMP to other compounds suggests that the metabolites of NMP, rather than NMP itself, may be the most suitable compounds for biological monitoring.

No methods for assessing exposure to NMP using a metabolite of NMP have been described so far. In our study, we report a method for the biological monitoring of NMP exposure using the concentration of 5-HNMP in plasma and the excretion of 5-HNMP in urine.

\section{Subjects and methods}

\section{Subjects and study design}

Six healthy male volunteers [mean age 35 (range 28 41) years, mean weight 74 (range 62-80) kg, and mean height 179 (range $170-186$ ) $\mathrm{cm}$ ] were studied. All the participants were subject to a general health examination, with special attention to liver, kidney, and hematological conditions. None of the subjects were using any kind of drug at the time of the experiment, and none had consumed alcohol within 24 hours of the experiment or as long as urine samples were collected or blood samples were sampled. There were no dietary restrictions before or after the exposure. During the exposure all the subjects at all 4 exposure levels had the same diet (after 2 hours, coffee and 2 slices of bread and cheese; after 4 hours, 200 grams of pizza; after 6 hours, coffee and 1 piece of cake; water as desired). The Ethics Committee of the Medical Faculty, Lund University, approved the study design, and all 6 subjects gave their written, informed consent to participate in the study.

\section{Exposure}

The volunteers were exposed in an exposure chamber with a turnover rate of 20 times per hour. The subjects were exposed 2 at a time. There was an exposure-free period of about 5 minutes after 2, 4, and 6 hours of exposure for the examination and biological sample collection. The exposure was determined by 4 consecutive 2 hour sampling periods in the personal breathing zone of each subject. The sampling was performed on Amberlite XAD-7 tubes (lot no 754; SKC pump, model 222). The 3 exposure levels (8-hour time-weighted average) were means of 10 (range $8-13$ ) $\mathrm{mg} / \mathrm{m}^{3}, 24$ (range 22-26) $\mathrm{mg} / \mathrm{m}^{3}$, and 53 (range $44-60$ ) $\mathrm{mg} / \mathrm{m}^{3}$. The generation of NMP air concentration and the analysis of the NMP air samples have been described earlier (2). There was at least 2 full weeks between the experimental exposures.

\section{Blood and urine samples for the toxicokinetics study}

Blood samples $(20 \mathrm{ml})$ were collected by venepuncture in evacuated heparinized tubes (Venoject, Teruma Europe NV, Leuven, Belgium) prior to, and at 4,8 (end of exposure), 9, 10, 12, 16, 24, 32, and 48 hours after the start of the exposure. After 30 minutes at room temperature the blood samples were centrifuged (1500 grams, 15 minutes), and the plasma was frozen and kept at $-16^{\circ} \mathrm{C}$ until the analysis. Urine was collected in polyethylene bottles before the exposure, at 2-hour intervals up to 16 hours after the start of the exposure, and then at 34 -hour and 38 -hour intervals up to 52 hours. The urine samples were frozen immediately and kept at $-16^{\circ} \mathrm{C}$ until the analysis.

\section{Analysis of 5-hydroxy-N-methyl-2-pyrrolidone in plasma and urine samples}

The method for determining 5-HNMP in urine (13) and plasma (14) has been described earlier. 5-HNMP was purified from the urine and plasma by adsorption to $\mathrm{a}_{8}$ solid phase extraction column and then eluted by ethyl acetate:methanol (80:20). After evaporation, the samples were derivatized at $100^{\circ} \mathrm{C}$ for 1 hour by bis(trimethylsilyl)trifluoroacetamide. Ethyl acetate was then added, and the samples were analyzed on a gas chromatograph (GC model 8065, Carlo-Erba, Milan, Italy) connected with a mass spectrometer (VG Trio 1000 quadrupole, Fisons, Manchester, UK) in the electron impact mode. The detection limit was $2 \mu \mathrm{mol} / 1$ for urine and $0.1 \mu \mathrm{mol} / \mathrm{l}$ for plasma.

\section{Results}

After the onset of the exposure to NMP, the hydroxylized compound (5-HNMP) appeared in plasma and urine. 
The mean 5-HNMP plasma concentrations [P-(5HNMP)] (figure 1] at the end of the exposure to 10,25 , and $50 \mathrm{mg} / \mathrm{m}^{3}$ were 8.0 (range $6.9-10.6$ ), 19.6 (range $16.0-23.6$ ), and 44.4(range 36.2-51.8) $\mu \mathrm{mol} / 1$, respectively. After the end of the exposure the P-(5-HNMP) still increased during the following 1-hour period and then decreased.

The 5-HNMP in the plasma elimination curves suggested a linear pattern (figure 2). An evaluation of the elimination by linear regression of the semilogarithmic concentration-time curves between 3 and 32 hours after the end of the exposure showed mean half-times of 5HNMP in plasma of 6.2 (range 5.3-6.8), 6.5 (range $5.8-7.5$ ), and 6.1 (range $5.4-7.1$ ) hours for the exposure levels 10,25 , and $50 \mathrm{mg} / \mathrm{m}^{3}$, respectively.

\section{P-5-HNMP $(\mu \mathrm{mol} / \mathrm{l})$}

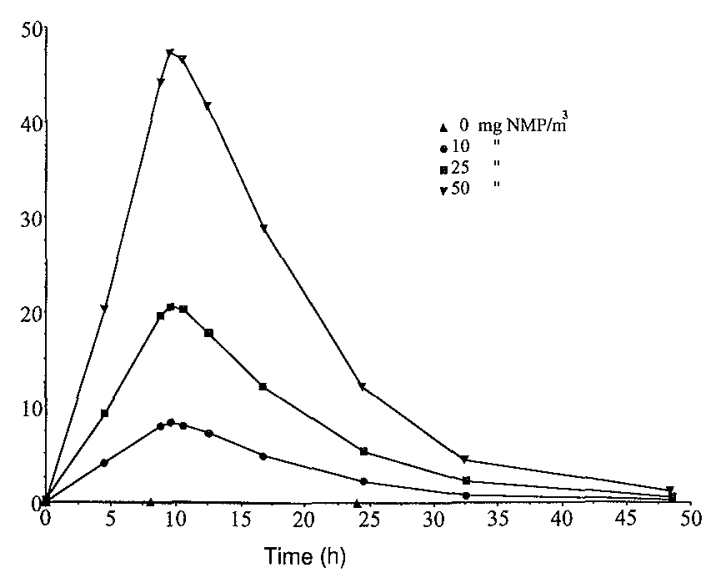

Figure 1. Mean concentration of 5-hydroxy- $\mathrm{N}$-methyl-2-pyrrolidone in plasma (P-5-HNMP) before, during, and after the exposure of 6 human volunteers to $\mathrm{N}$-methyl-2-pyrrolidone (NMP) concentrations of $0,10,25$, and $50 \mathrm{mg} / \mathrm{m}^{3}$.

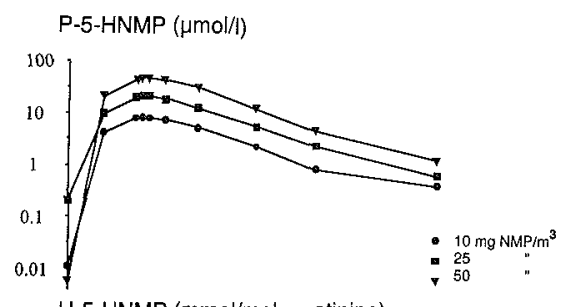

U-5-HNMP (mmol/mol creatinine)

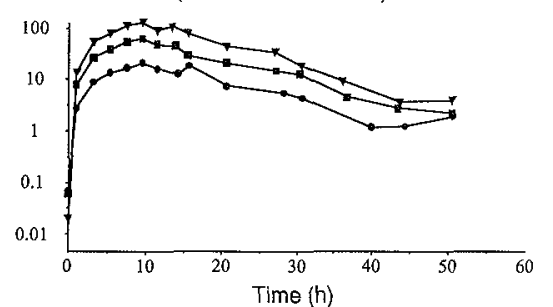

Figure 2. Mean concentrations of 5-hydroxy-N-methyl-2-pyrrolidone in urine (U-5-HNMP, corrected for creatinine concentration) and in plasma (P-5-HNMP) during and after the exposure of 6 male subjects to $\mathrm{N}$-methyl-2-pyrrolidone (NMP) concentrations of 10,25 , and 50 $\mathrm{mg} / \mathrm{m}^{3}$.
The concentration of 5-HNMP in urine [U-(5HNMP)] collected before the start of the NMP exposure and during the 0-exposure experiment was below the detection limit of $2 \mu \mathrm{g} / \mathrm{l}$. The U-(5-HNMP) rose during the exposure and during the following 2-hour period after the end of the exposure. Two hours after the end of the exposure the U-(5-HNMP) decreased, displaying a slight irregular pattern (figure 3). The irregular pattern was not fully eliminated by correction for the concentration of creatinine (figure 4) or for density. There was a very close correlation between U-(5-HNMP) corrected for creatinine and the excretion rate (correlation coefficients of 1.0 for all 3 exposure levels).

The mean U-(5-HNMP) in urine sampled during the last 2-hour period of exposure was 190 (range 140-250,

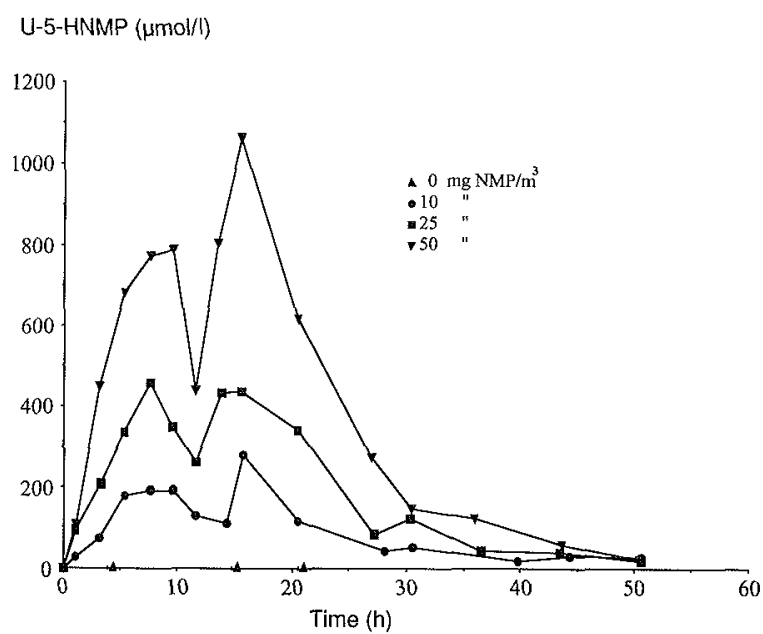

Figure 3. Mean concentration of 5-hydroxy-N-methyl-2-pyrrolidone (5-HNMP) in urine before, during, and after the exposure of 6 male subjects to $\mathrm{N}$-methyl-2-pyrrolidone (NMP) concentrations of $0,10,25$, and $50 \mathrm{mg} / \mathrm{m}^{3}$.

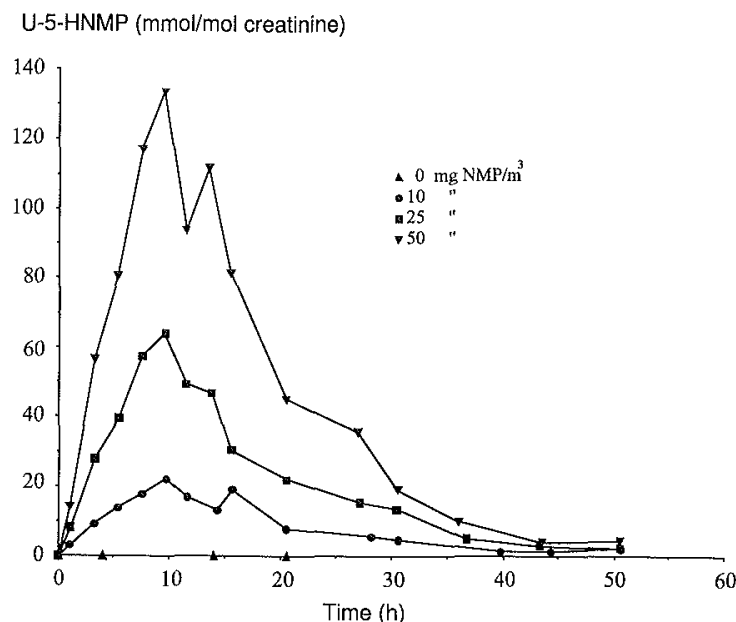

Figure 4. Mean concentration of 5-hydroxy-N-methyl-2-pyrrolidone in urine (U-5-HNMP, corrected for creatinine concentration) before, during, and after the exposure of 6 male subjects to $\mathrm{N}$-methyl-2pyrrolidone (NMP) concentrations of $0,10,25$, and $50 \mathrm{mg} / \mathrm{m}^{3}$. 
460 (range $200-840$ ), and 775 (range $490-1400$ ) $\mu \mathrm{g} / \mathrm{l}$ for the exposure levels of 10,25 , and $50 \mathrm{mg} / \mathrm{m}^{3}$, respectively (figure 3 ). The corresponding creatinine-corrected means of the U-(5-HNMP) concentrations were 17.7 (range 8.0-29.3), 57.3 (range 43.9-77.8), and 117 (range $83.1-146$ ) $\mathrm{mmol} / \mathrm{mol}$ creatinine, respectively (figure 4). There was a close correlation between the concentration of 5-HNMP in plasma at the end of the exposure and the 5-HNMP excretion in urine during the last 2-hours of the sampling period [correlation coefficient (r) 0.97$]$.

An evaluation of the mean excretion corrected for creatinine concentration by linear regression of the semilogarithmic concentration-time curves between 4 and 40 hours after the end of the exposure showed half-times of $6.7,8.2$, and 6.9 hours for the exposure levels of 10,25 , and $50 \mathrm{mg} / \mathrm{m}^{3}$, respectively (figure 2 ).

The amount of U-(5-HNMP) excreted in the urine collected during the exposure and 44 hours after the end of the exposure corresponded to 52 (range 43-66)\%, 62 (range $57-70$ ) \%, and 59 (range $41-74$ ) \% of the absorbed dose for the exposure levels of 10,25 , and 50 $\mathrm{mg} / \mathrm{m}^{3}$, respectively. The mean absorbed dose was calculated at an estimated pulmonary ventilation rate of 6 $\mathrm{m}^{3}$, a retention factor determined at the $50 \mathrm{mg} / \mathrm{m}^{3}$ exposure level of 0.90 (range $0.88-0.93$ ), and the inhaled NMP air concentration.

There was a close correlation between the 5-HNMP concentration in plasma $(\mathrm{r}=0.98)$ (figure 5) and the amount of U-(5-HNMP) excreted in the urine $(r=0.95)$ (figure 6) at the end of exposure versus the exposure to NMP. Individually, the linear relationship between 5HNMP at the end of the exposure in plasma and urine and the NMP exposure $\left(\mathrm{mg} / \mathrm{m}^{3}\right)$ was even closer. For plasma $(\mu \mathrm{mol} / \mathrm{l})$ the correlation coefficients were $>0.99$ with slopes from 0.67 to 1.00 (mean 0.84 ) and for urine

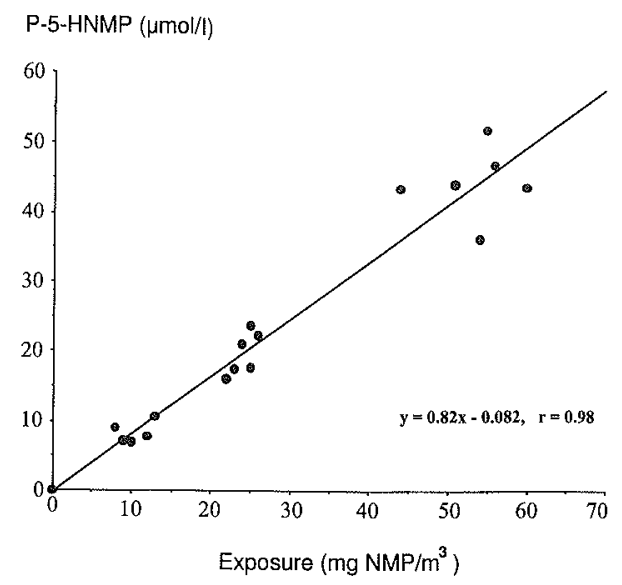

Figure 5. Concentration of 5-hydroxy- $\mathrm{N}$-methyl-2-pyrrolidone in plas$\mathrm{ma}(\mathrm{P}-5-\mathrm{HNMP})$ collected at the end of the exposure of 6 male subjects to $\mathrm{N}$-methyl-2-pyrrolidone (NMP) concentrations of $0,10,25$, and 50 $\mathrm{mg} / \mathrm{m}^{3}$.
( $\mathrm{mmol} / \mathrm{mol}$ creatinine) the corresponding value was $>0.98$ with slopes from 1.5 to 2.9 (mean 2.3 ).

\section{Discussion}

The present study displayed close correlations between the NMP air concentration and the 5-HNMP in plasma and urine. 5-HNMP is a major NMP metabolite with a half-time of about 6 hours in plasma. Thus, for assessing NMP exposure, 5-HNMP seems to be an excellent biomarker. Both 5-HNMP in plasma and in urine can be used.

Blood samples were collected up to 40 hours after the end of exposure. The highest 5-HNMP concentration in plasma [P-(5-HNMP)] was found in samples obtained 1 hour after the end of the exposure (figure 1). At the end of the studied period the P-(5-HNMP) was well above the detection limit for P-(5-HNMP). When calculated with a half-time of 6.3 hours and 1-compartment elimination, the P-(5-HNMP) at end of the study ( $6 \mathrm{x}$ halftime) were expected to be $0.13,0.32$, and $0.77 \mu \mathrm{mol} / 1$, respectively, for the 3 exposure levels. P-(5-HNMP) levels twice as high were found, however. This result may have been caused by a second, slower, compartment in the elimination phase, not observed due to the low P-(5HNMP) at the end of the studied period. In a study in which we administered NMP orally and collected urine during 9 days, we did not find a second phase of elimination (4). Moreover, the semilogarithmic P-(5-HNMP) concentration-time curves may display a slow uptake-distribution phase for 2 to 14 hours after exposure (figure 2). One explanation is the possibility of a concomitantly minor uptake through the skin, particularly if the absorption rate through the skin is low. Even though no aerosolization was observed during the exposure, based on the absence of condensation on cold surfaces, skin expo-

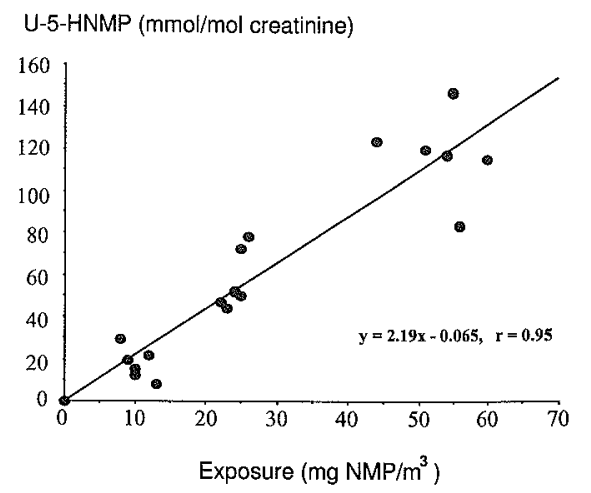

Figure 6. Concentration of 5-hydroxy-N-methyl-2-pyrrolidone in urine (U-5-HNMP, corrected for creatinine concentration) sampled during the 2 last hours of the exposure of 6 male subjects to $\mathrm{N}$-methyl-2pyrrolidone (NMP) concentrations of $0,10,25$, and $50 \mathrm{mg} / \mathrm{m}^{3}$. 
sure to NMP aerosol cannot be excluded. It should be noted that NMP may exist in various proportions of vapor and aerosol depending on the concentration, temperature and atmospheric humidity. The maximum vapor phase is $1320 \mathrm{mg} / \mathrm{m}^{3}$ at room temperature and dry air $(0 \%$ $\mathrm{RH}), 410 \mathrm{mg} / \mathrm{m}^{3}$ at normal humidity $(60 \% \mathrm{RH})$, and 0 $\mathrm{mg} / \mathrm{m}^{3}$ for wet air ( $100 \% \mathrm{RH}$ ) (unpublished data). There is, of course, also a possibility of uptake of gaseous NMP through the skin. Thus the calculated half-time in plasma may be somewhat different than the reported halftimes of $6-7$ hours. In a previous study with the oral administration of NMP in humans, a half-time of about 4 hours was roughly estimated (4). The recovered fraction of 5-HNMP in urine, $60 \%$, may also be overestimated if there is additional skin uptake. The importance of skin uptake has to be further investigated.

The elimination curve of U-(5-HNMP) showed a slight irregular pattern after the end of exposure. The irregularity was observed for all the subjects and at all the exposure levels. It may be caused by sample instability or analysis error. However, the stability of samples during prolonged storage has been checked, and the analysis method has been shown to display high reproducibility $(12,13)$. The irregular pattern of the elimination curve may be a result of skin uptake showing a second peak in the elimination curves about 4 hours after the first peak. The irregularity was not seen for plasma, but the sampling of plasma was less frequent than the urine sampling. The irregularity was not eliminated with correction for creatinine, density, or excretion rate. However, for biological monitoring, the irregularity seems to be of minor importance.

The major metabolite of NMP was 5-HNMP. The intraindividual difference in the hydroxylation of NMP to 5-HNMP was minor at the 3 studied exposure levels. Interindividually, the difference varied by a factor of 2 for urine. However, currently we have no knowledge of the toxicity of metabolites and, thus, whether small or large formations of 5-HNMP are favorable.

The close correlation between the NMP exposure level and the 5-HNMP concentration in plasma and urine offers several possible methods for biological monitoring. The use of 5-HNMP as the biomarker is favorable, as no risk of contamination of blood or urine may occur during the sampling. Because of the close correlation $(r=0.98)$ between the air level and the P-(5-HNMP) concentration at the end of exposure (figure 5) and the slight irregular elimination of U-(5-HNMP) (figure 4), we prefer plasma for biological monitoring. Exposure during 8 hours to an NMP air concentration of $10 \mathrm{mg} / \mathrm{m}^{3}$ during light work corresponds to a P-(5-HNMP) concentration of $8.2 \mu \mathrm{mol} / 1$ at the end of exposure. However, if nonvascular methods are preferable, the correlation between the NMP air level and U-(5-HNMP, $\mu \mathrm{mol} / \mathrm{mol}$ creatinine) or U-(5-HNMP, $\mu \mathrm{mol} /$ hour) showed correlation factors of 0.95 and 0.96 , respectively, for urine samples collected during the 2 last hours of exposure. U-(5-HNMP) of $22 \mu \mathrm{mol} / \mathrm{mol}$ creatinine and U-(5-HNMP) of $15 \mu \mathrm{mol} /$ hours corresponds to an airborne 8-hour NMP exposure of $10 \mathrm{mg} / \mathrm{m}^{3}$ during light work. Biological monitoring using the excretion rate for assessing the exposure is more difficult with urine sampling. Thus U-(5-HNMP) corrected for creatinine concentration is recommended for assessing the exposure to NMP when P-(5-HNMP) is not preferred.

The Swedish occupational exposure limit for NMP is $200 \mathrm{mg} / \mathrm{m}^{3}$. The corresponding limit in Germany is 90 $\mathrm{mg} / \mathrm{m}^{3}$. With the recommended method for biological monitoring it is possible to assess exposures far below 1 $\mathrm{mg} / \mathrm{m}^{3}$.

\section{Acknowledgments}

We thank Ms Karin Paulsson and Ms Kerstin Olsson for their skillful technical assistance. This work was supported by the Swedish Council for Work Life Research.

\section{References}

1. Åkesson B. N-methyl-2-pyrrolidone (NMP): nordic expert group for criteria documentation of health risks from chemicals. Stockholm: Arbetarskyddsverket, 1994:1-24. Arbete och Hälsa 40.

2. Åkesson B, Paulsson K. Experimental exposure of male volunteers to N-methyl-2-pyrrolidone (NMP): acute effects and pharmacokinetics of NMP in plasma and urine. Occup Environ Med 1997;54:236-40.

3. Wells D, Hawi AA, Digenis GA. Isolation and identification of the major metabolite of $\mathrm{N}$-methylpyrrolidone in the rat. Drug Metab Dispos 1992;21:124-6.

4. Åkesson B, Jönsson BAG. Major metabolic pathway for Nmethyl-2-pyrrolidone in humans. Drug Metab Dispos 1997;25:267-9.

5. Ursin C, Hansen CM, van Dyk JW, Jensen PO, Christensen IJ, Ebbehoej J. Permeability of commercial solvents through living human skin. Am Ind Hyg Assoc J 1995;56:651-60.

6. Priborsky J, Mühlbachova E. Evaluation of in vitro percutaneous absorption across human skin and in animal models. J Pharm Pharmacol 1990;42:468-72.

7. Midgley I, Hood AJ, Chasseaud LF, Brindley CJ, Baughman $S$, Allan G. Percutaneous absorption of co-administered N.methyl-2-[ $\left[{ }^{14} \mathrm{C}\right]$ pyrrolidinone and $2-\left[{ }^{14} \mathrm{C}\right]$ pyrrolidinone in the rats. Food Chem Toxicol 1992;30(1):57-64.

8. Lee KP, Chromey NC, Culik R, Barnes JR, Schneider PW, Toxicity of N-methyl-2-pyrrolidone (NMP): teratogenic, subchronic and two-year inhalation studies. Fundam Appl Toxicol 1987;9:222-35.

9. Beaulieau HJ. Schmerber KR. M-pyrol (NMP) use in the microelectronics industry. Appl Occup Environ Hyg $1991 ; 6: 874-80$. 
10. Leira HL, Tiltnes A, Svendsen K. Irritant cutaneous reactions to N-methyl-2-pyrrolidone NMP). Contact Dermatitis 1992;27:148-50

11. Hass U, Jakobsen BM, Lund SP. Effects of prenatal exposure to $\mathrm{N}$-methylpyrrolidone on postnatal developmental in rat. Pharmacol Toxicol 1995;76:406-9.

12. Solomon GM, Morse EP, Garbo MJ, Milton DK. Stillbirth after occupational exposure to $\mathrm{N}$-methyl-2-pyrrolidone. J Occup Environ Med 1996;38(7):705-13.
13. Jönsson BAG, Åkesson B. Determination of 5-hydroxy-Nmethylpyrrolidone and 2-hydroxy- $\mathrm{N}$-methylsuccinimide in human urine. J Chromatogr B 1997;694:351-7.

14. Jönsson BAG, Åkesson B. Analysis of 5-hydroxy-N-methyl2-pyrrolidone and 2-hydroxy-N-methylsuccinimide in plasma. Chromatographia 1997;46:141-4.

Received for publication: 3 February 1999 